\title{
Effects of Long-Term Regular Continuous and Intermittent Walking on Oxidative Stress, Metabolic Profile, Heart Rate Variability, and Blood Pressure in Older Adults with Hypertension
}

\author{
Piyapong Prasertsri ${ }^{1},{ }^{1,2}$ Jatuporn Phoemsapthawee ${ }^{(D)},{ }^{3}$ Sirigoon Kuamsub ${ }^{4},{ }^{4}$ \\ Kulwara Poolpol $(1),{ }^{1}$ and Orachorn Boonla $\mathbb{1}^{1,2}$ \\ ${ }^{1}$ Faculty of Allied Health Sciences, Burapha University, Saen Suk, Chonburi 20131, Thailand \\ ${ }^{2}$ Exercise and Nutrition Innovation and Sciences Research Unit, Burapha University, Saen Suk, Chonburi 20131, Thailand \\ ${ }^{3}$ Department of Sports Science and Health, Faculty of Sports Science, Kasetsart University, Nakhon Pathom 73140, Thailand \\ ${ }^{4}$ Faculty of Abhaibhubejhr Thai Traditional Medicine, Burapha University, Saen Suk, Chonburi 20131, Thailand
}

Correspondence should be addressed to Piyapong Prasertsri; piyapong@buu.ac.th

Received 29 June 2021; Revised 4 January 2022; Accepted 18 January 2022; Published 31 January 2022

Academic Editor: Linda M. Gerber

Copyright (c) 2022 Piyapong Prasertsri et al. This is an open access article distributed under the Creative Commons Attribution License, which permits unrestricted use, distribution, and reproduction in any medium, provided the original work is properly cited.

\begin{abstract}
It is documented that regular exercise is beneficial for improving the antioxidant system, metabolic system, cardiac autonomic function, and blood pressure in those with hypertension. In this regard, low-intensity exercise is recommended for older adults, particularly those with chronic diseases. This study aimed to compare the effects of long-term regular continuous walking with intermittent walking on oxidative stress, metabolic profile, heart rate variability, and blood pressure in older adults with hypertension. Forty-three participants with hypertension aged 60-80 years were randomly divided into the continuous or intermittent walking (CON or INT) groups. Participants in the CON group walked for $30 \mathrm{~min}, 3$ days/week for 12 weeks. Participants in the INT group split 30 min walking into 3 identical sessions punctuated by a 1 min rest after each session, 3 days/ week for 12 weeks. Antioxidant and oxidative stress markers, metabolic markers, heart rate variability, and blood pressure were evaluated before and after the exercise program. Glutathione (GSH), GSH to GSH disulfide (GSSG) ratio, and total GSH increased significantly, and GSSG and malondialdehyde decreased significantly in both groups $(p<0.05)$ without significant differences between groups. Triglycerides, ratio of total cholesterol to high-density lipoprotein cholesterol, and atherosclerogenic index were significantly lower in the CON group than those in the INT group $(p<0.05)$. The standard deviation of the NN intervals and root mean square of the successive differences were significantly higher, and low-frequency power was significantly lower in the INT group than that in the CON group $(p<0.05)$. No significant changes in blood pressure were noted in both groups, and nor were there any significant differences between groups. Long-term regular continuous and intermittent walking may comparably increase antioxidants, reduce oxidative stress, and be beneficial for improving important blood pressure-related outcomes, including metabolic profile or cardiac autonomic function in older adults with hypertension.
\end{abstract}

\section{Introduction}

Hypertension affects more than 1 billion people worldwide, and that number is growing [1], mainly as a result of an aging population [2]. In 2016, 17.9 million or $44 \%$ of noncommunicable disease-related deaths were due to cardiovascular disease (CVD) with hypertension the leading risk factor [3]. Hypertension is also the leading preventable risk factor for premature death and disability. Accordingly, treatment and control of hypertension are critically significant for preventing consequent CVD, premature death, and disability [4].

Extensive evidence of cardiovascular, metabolic, and autonomic nervous benefits has led many guidelines to suggest long-term regular exercise as one of the best proven nonpharmacological interventions for preventing and 
treating hypertension [5]. Long-term regular exercise is also associated with the boost of antioxidant capacity and diminution of oxidative stress levels, which subsequently results in redox balance conservation and cellular homeostasis [6]. Oxidative stress plays a mechanistic role in the control of blood pressure (BP) and the development of hypertension and CVD [6, 7].

Considering exercise for older adults, exercise characteristics, such as intensity, type, and mode, are essential factors that should be judiciously examined. Regarding intensity, low-intensity exercise is often recommended for older adults due to it being easier to perform, which makes it safe and feasible amid practice [8]. Whether an exercise is practiced continuously or intermittently could be another important factor that impacts its effects. Continuous aerobic exercise is defined as any activity using large muscle groups, including walking. Thus, it has been widely reported to produce positive effects on health, including the cardiovascular system [9]. In addition to continuous exercise, intermittent exercise is characterized by repeated short bouts of exercise separated by periods of rest [10], which may be more suited to older individuals with a sedentary lifestyle who are predisposed to a decline in aerobic capacity [11]. In recent years, intermittent exercise has gained a lot of attention for its time-efficient nature and beneficial effects on physical functions [8]. It has been suggested that intermittent exercise results in similar or better physiological outcomes than continuous exercise as a single bout [12]. The earliest studies of intermittent exercise noted that regular practice of this form of exercise is beneficial in terms of modulating vagal activity, adapting the cardiovascular system [13], reducing the risk of metabolic syndrome, and improving body composition [14].

Regarding data from the National Health and Nutrition Examination Surveys (NHANES), Fan and colleagues [15] revealed that the participation rate in walking was ranked first as a leisure time physical activity among all ages of American women. Previous studies reported that the systolic BP (SBP) level of participants with increased BP or essential hypertension reduced significantly by $2.6-21.3 \mathrm{mmHg}$ following regular walking for $12-24$ weeks $[16,17]$. In rats, walking also correlated positively with metabolic and hemodynamic changes [5]. It is well documented that oxidative stress, cardiovascular risk factors, and cardiac autonomic function are key factors influencing BP [18, 19]. Furthermore, little is known about these responses to low-intensity, continuous, and intermittent walking, particularly as a consequence of long-term practice and in older adults with hypertension. Accordingly, this study aimed to compare the effects of long-term regular continuous walking with intermittent walking primarily on oxidative stress and secondarily on metabolic profile, heart rate variability, and blood pressure in older adults with hypertension.

\section{Materials and Methods}

2.1. Study Design and Screening of Participants. This was a pretest-posttest designed experimental study. Fifty older adults in Mueang District, Chonburi Province, Thailand, were recruited from the Aging Society of Mueang District and the Aging Society of Burapha University Hospital, Chonburi Province, Thailand, from February to June 2018. To calculate the sample size, a statistical formula for comparing the mean read-outs of two groups of participants was applied following Hernández-Torres et al. [20]. Continuous and intermittent exercise increased high-density lipoprotein cholesterol (HDLC) to 39.5 and $45.5 \mathrm{mg} / \mathrm{dL}$, respectively, with a standard deviation of 12.7. With an $\alpha$ error of 0.05 and a $\beta$ error of 0.20 , the proposed sample size of this study was 25 participants per group with a total of 50 participants, including a $10 \%$ dropout rate.

Participants were invited to the laboratory and screened on the morning of the physical examination and history taking. The physical examination included BP and heart rate (HR) measurements. In addition, a health questionnaire form and interviews were taken to assess underlying diseases, drug treatment, history of illness, and prior drug treatment. Participants were enrolled in the study after screening, based on the inclusion and exclusion criteria and provision of informed consent. Hypertension was defined by the 2018 ESH/ESC Guidelines [21]. These guidelines classify hypertension as resting SBP of $\geq 140 \mathrm{mmHg}$ and/or diastolic $\mathrm{BP}$ (DBP) of $\geq 90 \mathrm{mmHg}$.

The inclusion criteria were (a) male or female; (b) age, 60-80 years; and (c) diagnosed with hypertension or SBP $\geq 140 \mathrm{mmHg}$ or DBP $\geq 90 \mathrm{mmHg}$. The exclusion criteria were (a) obesity, CVD, diabetes mellitus (DM), kidney disease, thyroid disease, or musculoskeletal disease; (b) regular exercise; and (c) regular smokers or drinkers. Consequently, the withdrawal criteria were (a) diagnosed with or received drugs to treat obesity, CVD, DM, kidney disease, thyroid disease, or musculoskeletal disease during participation in the study; (b) unable to practice exercise for at least $80 \%$ of the program; and (c) requesting to withdraw from the study.

Most of the participants received BP-lowering medications, such as calcium channel blockers, angiotensin receptor blockers, angiotensin converting enzyme inhibitors, beta-blockers, or diuretics. Eight participants were newly diagnosed and had not received any medications, and 42 were on treatment and reached their target BP (SBP $<140 \mathrm{mmHg}$ and DBP <90 mmHg).

This study was conducted under the approval of the Human Ethics Committee of Burapha University (approval no. 218/2560) and was in accordance with the ethical standards of the 2013 Declaration of Helsinki. This study is registered with the Thai Clinical Trials Registry (identification no. TCTR20180226003).

2.2. Study Protocols. One week after screening, each participant was invited to the laboratory in the morning to measure the outcomes and receive an exercise program. Each participant was randomized and allocated into continuous or intermittent walking group (CON or INT group). Participants in the CON group were instructed to walk at a rhythm of $1 \mathrm{step} / \mathrm{sec}$ or $60 \mathrm{steps} / \mathrm{min}$ for $30 \mathrm{~min}$ continuously, 3 days/week for 12 weeks. Participants in the INT group were instructed to split $30 \mathrm{~min}$ walking into equal 3 
sessions with the same rhythm as the CON group, punctuated by a $1 \mathrm{~min}$ rest after each session, 3 days/week for 12 weeks.

The exercise program was designed as a home-based exercise. Participants were demonstrated and practiced walking correctly with a metronome obtained from a mobile application (Pro Metronome, EUMLab, Xanin Technology $\mathrm{GmbH}$ ) under the supervision of a researcher prior to performing the exercise at home. Participants in both groups were asked to maintain their daily dietary intake and routine activities, such as housekeeping, gardening, and cooking without any additional regular exercise, that is, walking for 12 weeks. Participants who could not complete the program (i.e., those exhibiting a health problem or requesting to quit the study) were asked to inform the researcher as soon as possible. Adherence to and compliance with the exercise program were recorded for each participant. Participants were also asked to record their exercise schedule during the study period on the record sheet provided by a researcher. Participants' exercise was closely followed weekly via telephone by a researcher or research assistant. Oxidative stress, metabolic factors, HR variability (HRV), and BP were assessed before and after the exercise program.

2.3. Antioxidant and Oxidative Stress Assays. Glutathione (GSH) is an endogenous antioxidant involved in many signaling pathways, including modification of the redox potential toward oxidative values and formation of the disulfide bond between protein thiol groups [22]. GSH, GSH disulfide (GSSG), and total GSH in this study were measured in whole blood as described previously by Nakmareong et al. [23]. The GSH/GSSG redox ratio was obtained by calculation. Malondialdehyde (MDA), a biomarker of lipid peroxidation [24], was determined in plasma using thiobarbituric acid as previously described by Kukongviriyapan et al. [25].

2.4. Biochemical Assay. Plasma glucose and serum triglyceride (TG) and total cholesterol (TC) concentrations were measured by enzymatic methods. Serum HDLC concentration was measured with the accelerator selective detergent method. Serum low-density lipoprotein cholesterol (LDLC) concentration was obtained by calculation. These assays were achieved using a standard automated laboratory machine (Architect c8000, Abbott, Lake Bluff, IL, USA). Atherosclerosis is a chronic inflammatory disease of the arteries, associated with blood lipid disorder and oxidative stress [6]. This study also determined the atherosclerogenic index (AI) as an indicator of coronary heart disease and metabolic risk calculated from the following equation: $\mathrm{AI}=(\mathrm{TC}-\mathrm{HDLC}) / \mathrm{HDLC}$.

The participants' venous blood was obtained from an antecubital vein following a 12-hour overnight fast and not taking medications and collected in glucose, EDTA, and clot activator tubes. All analyses were executed within 2 hours after collection.

2.5. Blood Pressure and Cardiac Autonomic Function Assessment. Following a 12-hour overnight fast and not taking medications, the participant's $\mathrm{BP}$ was measured in the supine position after resting for $15 \mathrm{~min}$, using a digital automatic BP monitor (Microlife BP 3AQ1, Widnau, Switzerland). BP was measured 3 times, 5 min apart, and the average of the three readings was reported. Pulse pressure (PP: SBP - DBP), mean arterial pressure (MAP: DBP + (PP/ $3)$ ), and rate-pressure product ( $\mathrm{RPP}: \mathrm{SBP} \times \mathrm{HR}$ ) were further calculated from the SBP, DBP, and HR.

HRV, according to a short-term analysis, was used to assess cardiac autonomic function. Lead II electrocardiography (PowerLab 4/30, AD Instruments, Bella Vista, NSW, Australia) was applied to record and analyze the HRV data. Participants' HRV data were collected for 15 min subsequent to the BP measurement. Analysis of HRV data incorporated the time and frequency domains. The time domain consisted of the SDNN and RMSSD values. The frequency domain was comprised of the values of total power (TP), very low, low, and high-frequency powers (VLF: DC to $0.04 \mathrm{~Hz}, \mathrm{LF}: 0.04-0.15 \mathrm{~Hz}$, and HF: $0.15-0.4 \mathrm{~Hz}$ ), and the $\mathrm{LF} / \mathrm{HF}$ ratio.

\subsection{Anthropometry and Body Composition Measurement.} Participant height was measured using a stadiometer (Health-O-Meter ProSeries, Pelstar Inc., New York, NY, USA) during inspiration. Fat distribution was measured from waist and hip circumferences and their ratio. Waist circumference (WC) was measured at the end of a normal expiration and at the midpoint between the bottom rib and the superior iliac spine. Hip circumference (HC) was measured on a horizontal plane at the level of maximum buttock extension. Body mass (BM), body mass index (BMI), and body composition (percent body fat, fat mass, fat-free mass, protein mass, mineral mass, and water mass) were measured while wearing minimal clothing using a body composition analyzer (InBody270, InBody Co. Ltd., Deajeon, Korea) based on the principle of bioelectrical impedance analysis. All measurements were taken in the standing position.

2.7. Data Analyses. Data normality was analyzed and confirmed using the Shapiro-Wilk test. Two-way repeated measures analysis of variance (with Bonferroni post hoc test) was performed to detect differences in variables between the groups (CON and INT groups) after the exercise program and in each group before and after the exercise program. Fvalues determining whether rejection of the null hypothesis and Cohen's d effect size are also reported in case of having a significant difference between groups. The independent $t$ test was applied to analyze the differences between groups before the exercise program. All analyses were carried out using IBM SPSS Statistics software (IBM Corp., Armonk, NY, USA). Data are shown as mean \pm SD. A $p$ value $<0.05$ was considered significant.

\section{Results and Discussion}

Forty-three of 50 participants (86\%) completed the study. Seven participants, including 3 participants in the CON group (12\%) and 4 participants in the INT group (16\%), 
requested to stop participating in the study as they could not follow the exercise program. Thus, data from 43 participants (22 participants in the CON group and 21 participants in the INT group) were analyzed and reported in the results.

3.1. Physical and Physiological Characteristics. No significant differences were observed in physical, except for gender, or physiological characteristics, including age, height, BM, $\mathrm{BMI}$, percent body fat $(\% \mathrm{BF})$, fat mass $(\mathrm{FM})$, percent fat-free mass (\%FFM), FFM, percent body water (\%body water), water mass, protein mass, mineral mass, visceral fat (VF) level, WC, HC, or the $\mathrm{W} / \mathrm{H}$ ratio between the CON and INT groups before and after the exercise program.

Within-group comparisons demonstrated that $\mathrm{HC}$ decreased significantly $(p=0.047)$ in the CON group following the exercise program. \%BF $(p=0.026)$, FM $(p=0.037)$, HC $(p=0.024)$, and VF level $(p=0.030)$ decreased significantly, and \%FFM $(p=0.024)$ and \%body water $(p=0.026)$ increased significantly in the INT group following the exercise program (Table 1).

\subsection{Antioxidant and Oxidative Stress Markers.} Antioxidant markers, including blood total GSH, GSH, and GSSG concentrations, the GSH/GSSG ratio, and oxidative stress marker, including plasma MDA concentration, were not different between the CON and INT groups before and after the exercise program.

In both the CON and INT groups, blood total GSH $(p=0.023$ and $p \leq 0.001)$ and GSH $(p=0.022$ and $p \leq 0.001)$ concentrations and the GSH/GSSG ratio ( $p=0.014$ and $p=0.002$ ) increased significantly, and blood GSSG $(p \leq 0.001$ and $p=0.002)$ and plasma MDA $(p=0.023$ and $p \leq 0.001)$ concentrations decreased significantly following the 12-week exercise program (Figures 1-4).

3.3. Metabolic Profile. No significant differences were observed in the metabolic variables, including the concentrations of plasma glucose and serum TG, TC, HDLC, and LDLC, the TC/HDLC ratio, and AI between the CON and INT groups before the exercise program. Moreover, TG concentration $(p=0.017, F=6.224$, and effect size $=0.135)$, the TC/HDLC ratio $(p=0.048, F=4.151$, and effect size $=0.094)$, and AI $(p=0.048, F=4.151$, and effect size $=0.094)$ were significantly lower in the CON group than those in the INT group after the exercise program.

HDLC concentration increased significantly $(p=0.015)$, and TG concentration $(p=0.011)$, the TC/HDLC ratio $(p=0.003)$, and AI $(p=0.003)$ decreased significantly within the CON group after the exercise program. In addition, plasma glucose concentration decreased significantly $(p=0.011)$ within the INT group after the exercise program (Table 2).

3.4. Heart Rate and Heart Rate Variability. No significant differences were observed in HR and HRV variables, including SDNN, RMSSD, TP, VLF power, LF power, HF power, or the LF/HF ratio between the groups before the exercise program. Moreover, SDNN $(p=0.016, F=6.272$, and effect size $=0.136)$ and RMSSD $(p=0.018, F=6.107$, and effect size $=0.132$ ) were significantly higher, and $\mathrm{HR}$ $(p=0.014, F=6.627$, and effect size $=0.142)$ and LF power in the normalized unit (LFnu power) $(p=0.043, F=4.362$, and effect size $=0.098$ ) were significantly lower in the INT group than those in the CON group after the exercise program.

SDNN $(p=0.028)$ and RMSSD $(p=0.047)$ decreased significantly within the CON group following the exercise program. Besides, HR $(p=0.006)$ and LFnu power ( $p=0.006)$ decreased significantly, and HF power in the normalized unit (HFnu power) closely reached significance $(p=0.050)$ within the INT group after the exercise program (Table 3).

3.5. Blood Pressure. BP variables, including SBP, DBP, PP, $\mathrm{MAP}$, and RPP, were not different between the groups before the exercise program. However, RPP $(p=0.049, F=4.123$, and effect size $=0.093$ ) was significantly lower in the INT group than that in the CON group after the exercise program.

The BP variables were not different within the CON group, whereas $\operatorname{PP}(p=0.037)$ and $\operatorname{RPP}(p=0.003)$ decreased significantly within the INT group following the exercise program (Table 3 ).

While most hypertension guidelines recommend moderate-to-high-intensity exercise, middle-aged or older adults performing exercise at moderate or high intensity sometimes encounter maladaptation problems, such as exerciseinduced joint pain, prolonged palpitation/arrhythmia, chest tightness or dyspnea, and even ischemic events that hinder them from exercising further [26]. Thus, we aimed to explore a low yet effective intensity of exercise to add to the management of hypertension. The primary goal of this study was to establish a practical exercise program that older adults with hypertension could engage in at their leisure with less fatigue and breathlessness, yet provided health benefits. Participants in the two groups walked at a relatively slow speed. They were encouraged to walk at a rhythm of 1 step/ $\mathrm{sec}$ or $60 \mathrm{steps} / \mathrm{min}$ and prescribed a similar $30 \mathrm{~min} /$ day volume of exercise. As described by Hall et al. [27] who measured the metabolic cost of walking activities in older adults, the intensity of walking exercise in the present study was classified as low-intensity exercise. Our walking exercise was relatively comparable to slow walking $(<3 \mathrm{~km} / \mathrm{h})$ at approximately 2.0 metabolic equivalents (METs) [28].

Although walking is defined as low-intensity exercise, this study separated $30 \mathrm{~min}$ walking exercise periods into 3 identical sessions (10 min/session), aimed at safer, more feasible practice. In addition, this study included older participants with a sedentary lifestyle (not regular exercisers) exhibiting a decline in aerobic capacity [11]. Thus, partaking in short periods of rest may be more suited to their physical fitness. Moreover, the punctuation of the $10 \mathrm{~min}$ walking exercise by a brief 1 min rest (work : rest ratio $=10: 1$ ) was administered to sustain mitochondrial responses mediated 
TABle 1: Physical and physiological characteristics of participants before and after the exercise program.

\begin{tabular}{|c|c|c|c|c|c|}
\hline & \multicolumn{2}{|c|}{ CON group $(n=22)$} & \multicolumn{2}{|c|}{ INT group $(n=21)$} & \multirow{2}{*}{$p$ value } \\
\hline & Before & After & Before & After & \\
\hline Age (yrs) & $70.95 \pm 5.00$ & $71.20 \pm 5.00$ & $70.86 \pm 6.51$ & $71.11 \pm 6.51$ & 0.956 \\
\hline Gender (M/F) (\%) & $8 / 14(36 / 64)$ & $8 / 14(36 / 64)$ & $2 / 19(10 / 90)$ & $2 / 19(10 / 90)$ & 0.037 \\
\hline Height $(\mathrm{m})$ & $1.57 \pm 0.08$ & $1.57 \pm 0.08$ & $1.55 \pm 0.07$ & $1.55 \pm 0.07$ & 0.524 \\
\hline Body mass (kg) & $56.65 \pm 10.72$ & $56.66 \pm 10.57$ & $59.33 \pm 8.11$ & $57.07 \pm 10.66$ & 0.592 \\
\hline BMI $\left(\mathrm{kg} / \mathrm{m}^{2}\right)$ & $22.97 \pm 3.54$ & $22.93 \pm 3.59$ & $24.55 \pm 2.66$ & $22.95 \pm 3.53$ & 0.369 \\
\hline Body fat (\%) & $29.71 \pm 8.09$ & $29.68 \pm 8.32$ & $36.65 \pm 6.36$ & $30.84 \pm 7.59^{*}$ & 0.334 \\
\hline Fat mass $(\mathrm{kg})$ & $17.03 \pm 6.25$ & $17.04 \pm 6.33$ & $21.83 \pm 5.13$ & $17.76 \pm 5.92^{*}$ & 0.300 \\
\hline Fat-free mass (\%) & $70.29 \pm 8.11$ & $70.33 \pm 8.33$ & $63.29 \pm 6.31$ & $69.17 \pm 7.60^{*}$ & 0.317 \\
\hline Fat-free mass (kg) & $39.61 \pm 7.79$ & $39.62 \pm 7.69$ & $37.46 \pm 5.92$ & $39.31 \pm 7.84$ & 0.517 \\
\hline Body water (\%) & $51.77 \pm 6.08$ & $51.79 \pm 6.21$ & $46.59 \pm 4.73$ & $50.90 \pm 5.64^{*}$ & 0.328 \\
\hline Water mass (kg) & $29.18 \pm 5.77$ & $29.18 \pm 5.69$ & $27.59 \pm 4.45$ & $28.93 \pm 5.80$ & 0.541 \\
\hline Protein mass (kg) & $7.74 \pm 1.55$ & $7.73 \pm 1.53$ & $7.24 \pm 1.16$ & $7.67 \pm 1.55$ & 0.434 \\
\hline Mineral mass (kg) & $2.70 \pm 0.49$ & $2.71 \pm 0.48$ & $2.68 \pm 0.40$ & $2.71 \pm 0.49$ & 0.873 \\
\hline Visceral fat level & $6.91 \pm 2.49$ & $6.95 \pm 2.50$ & $8.71 \pm 1.82$ & $7.24 \pm 2.28^{*}$ & 0.176 \\
\hline Waist circumference $(\mathrm{cm})$ & $82.30 \pm 11.52$ & $81.07 \pm 11.52$ & $85.54 \pm 7.77$ & $81.10 \pm 11.60$ & 0.473 \\
\hline Hip circumference $(\mathrm{cm})$ & $94.01 \pm 9.04$ & $92.72 \pm 7.89^{*}$ & $98.50 \pm 5.78$ & $93.33 \pm 7.61^{*}$ & 0.333 \\
\hline $\mathrm{W} / \mathrm{H}$ ratio & $0.88 \pm 0.06$ & $0.88 \pm 0.06$ & $0.89 \pm 0.06$ & $0.88 \pm 0.05$ & 0.989 \\
\hline
\end{tabular}

Data are mean \pm SD. CON, continuous walking; INT, intermittent walking; BMI, body mass index; W/H, waist to hip circumference ratio. ${ }^{*} p<0.05$, significantly different from before exercise program (two-way ANOVA).

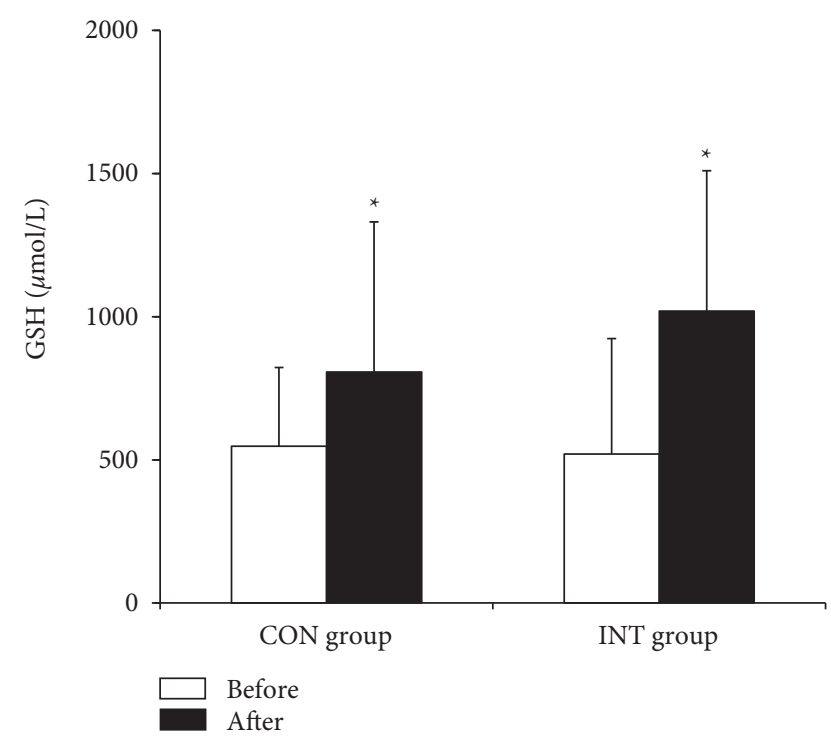

Figure 1: Blood glutathione (GSH) concentration in the continuous and intermittent walking (CON and INT) groups before and after the exercise program. Data are mean $\pm \mathrm{SD} .{ }^{*} p<0.05$, significantly different from before the exercise program (two-way ANOVA).

during the $10 \mathrm{~min}$ walking [29]. Furthermore, the $1 \mathrm{~min}$ rest duration was set concerning participants' fitness level, thereby making this form of walking a suitable option for older individuals with a sedentary lifestyle or with some functional limitations, that is, hypertension, heart diseases, DM, obesity, lung diseases, or musculoskeletal diseases.

3.6. Regular Low-Intensity Exercise and Oxidative Stress. To date, studies that have compared continuous to intermittent low-intensity exercise are limited. In this study,

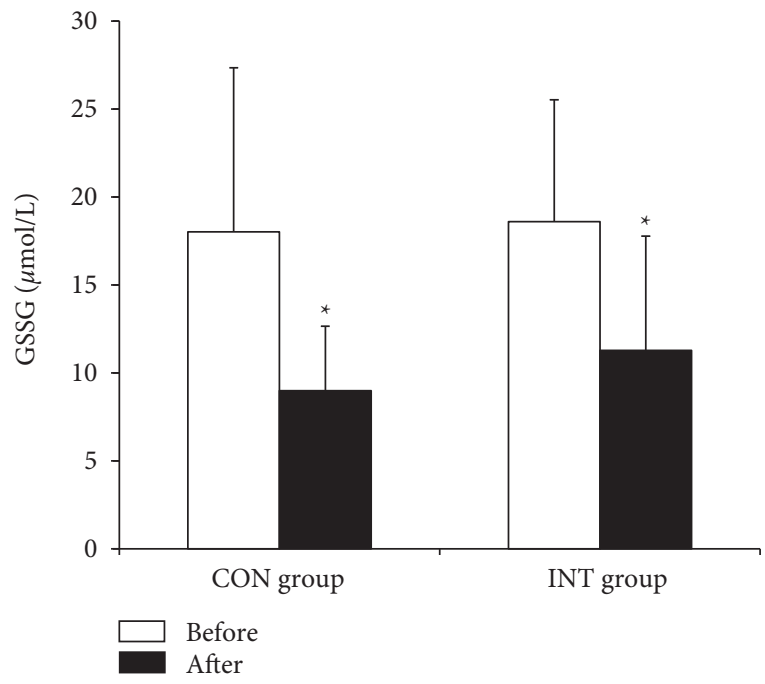

FIGURE 2: Blood glutathione disulfide (GSSG) concentration in the continuous and intermittent walking (CON and INT) groups before and after the exercise program. Data are mean \pm SD. ${ }^{*} p<0.05$, significantly different from before the exercise program (two-way ANOVA).

long-term regular continuous and intermittent walking was demonstrated to reduce oxidative stress by the increasing number of antioxidant enzymes, thus reinforcing the role of regular low-intensity exercise on the improvement of oxidative stress in physiological and pathological conditions. According to the free radical theory of aging, an accumulation of oxidative damage occurs with aging. This damage is linked to the principal cause of age-related declines in cellular function [30] as well as the development of agerelated diseases, including arthritis, DM, dementia, cancer, atherosclerosis, CVD, obesity, osteoporosis, metabolic syndrome, and hypertension [31, 32]. When considering 


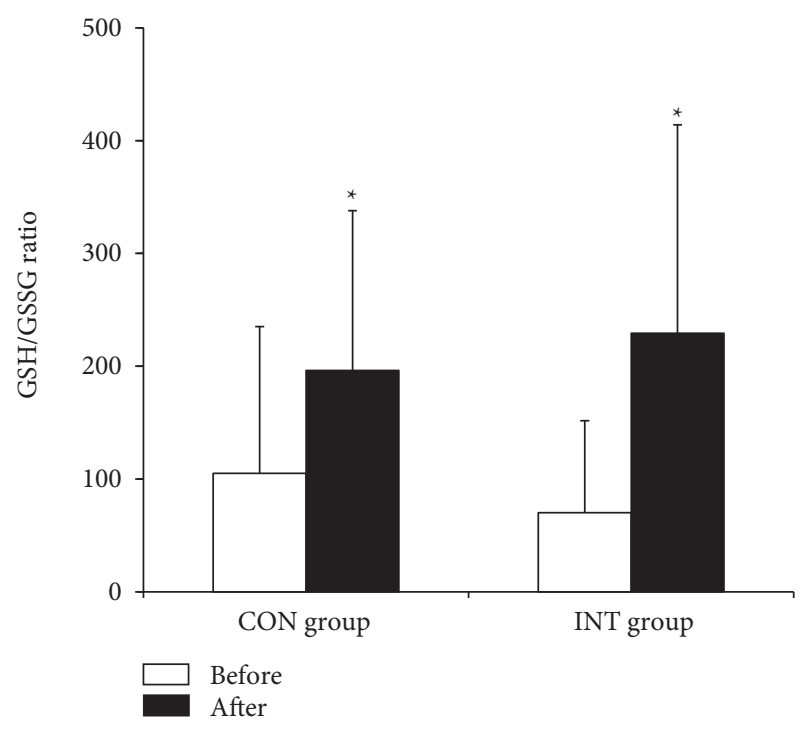

FIGURE 3: Glutathione to glutathione disulfide (GSH/GSSG) ratio in the continuous and intermittent walking (CON and INT) groups before and after the exercise program. Data are mean \pm SD. ${ }^{*} p<0.05$, significantly different from before the exercise program (two-way ANOVA).

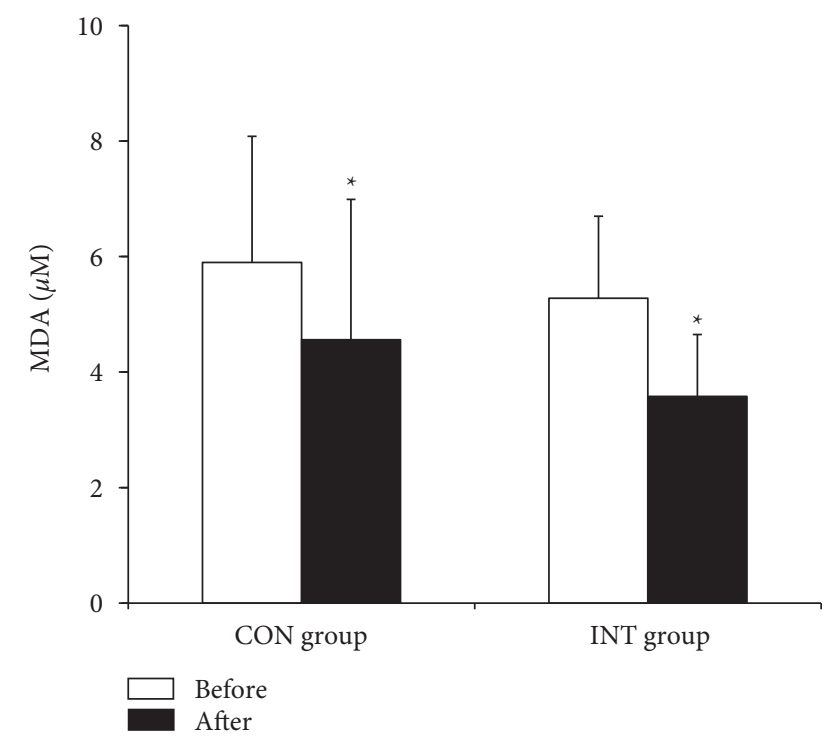

FIGURE 4: Plasma malondialdehyde (MDA) concentration in the continuous and intermittent walking (CON and INT) groups before and after the exercise program. Data are mean \pm SD . ${ }^{*} p<0.05$, significantly different from before the exercise program (two-way ANOVA).

hypertension, chronic inflammation may play a role in the pathogenesis of hypertension by the progression of arterial stiffness [33]. It is involved in high levels of free radicals inducing oxidative stress [34].

It has been proved that regular exercise exerts a vital role in alleviating oxidative stress, improving oxidative metabolism, and reducing inflammation [35]. Regular exercise effectively improves antioxidant capacity in the skeletal muscles, as evidenced by the increased activity of glutathione peroxidase and superoxide dismutase posttraining [36].

The present findings reveal that antioxidants increased significantly and oxidative stress decreased significantly in both hypertensive groups practicing continuous and intermittent walking for 12 weeks. This may reflect the mobilization and synthesis of the antioxidant systems in response to long-term regular exercise. The increase in GSH levels with long-term regular exercise in our study is in congruence with preceding findings [37,38]. A previously related study by Elokda and Nielsen [38] demonstrated an increased GSH and GSH/GSSG ratio in addition to decreased GSSG levels in healthy sedentary volunteers after regular exercise simulating outpatient cardiac rehabilitation throughout 6 weeks. In adaptation to incremental exercise, Bouzid et al. [39] found that GPx and $\alpha$-tocopherol levels increased significantly in older adults who performed regular low-intensity aerobic exercise.

Mechanisms underlying increased GSH antioxidants after regular exercise, including low-intensity exercise, have previously been described, for instance, upregulation of endogenous antioxidant activity [39, 40]. Besides that, the expression of inflammatory cytokines such as tumor necrosis factor- $\alpha$, which has been revealed to impair intracellular levels of the important cofactor of GPx, is attenuated following regular exercise in chronic heart failure patients [41]. This might be linked to increased concentrations of blood GSH.

In addition to improving antioxidant defense, regular exercise alleviates lipid peroxidation both in adults and in aged individuals [42]. As previously reported in rats, 12 weeks of regular low-intensity cardiovascular exercise reduced oxidative stress and augmented nitric oxide (NO) bioavailability, thus allowing improvement in coronary artery endothelial dysfunction in rats with hypertension [43]. Klarod et al. [44] reported on healthy young adults who performed 8 weeks of regular low-intensity cycling-based exercise, demonstrating that MDA levels decreased significantly. This report further suggested that regular submaximal exercise may prevent lipid peroxidation through lactate metabolism and changes in the NADH/NADPH ratio, which are linked to the tissue adaptive response to exercise. Enhanced lactate uptake through submaximal exercise increases NADH/NADPH ratio, which promotes antioxidant enzyme activity to scavenge free radicals [44].

3.7. Regular Low-Intensity Exercise and Heart Rate Variability. The present study also observed an improvement in cardiac autonomic function measured by HRV. HRV is of interest, as a wide range of diseases are associated with decreased variability, including hypertension, DM, CVD, and psychiatric disorders [45]. In this study, SDNN and RMSSD values were significantly greater, and LF power value was significantly lower in participants participating in intermittent walking. These changes were accompanied by a significantly lower HR ( -3.4 beats $/ \mathrm{min})$. This negative chronotropic effect on HR may be related to an increase in vagal drive as revealed by time domain measures of HRV, 
TABLe 2: Metabolic profile of participants before and after the exercise program.

\begin{tabular}{|c|c|c|c|c|c|}
\hline & \multicolumn{2}{|c|}{ CON group $(n=22)$} & \multicolumn{2}{|c|}{ INT group $(n=21)$} & \multirow{2}{*}{$p$ value } \\
\hline & Before & After & Before & After & \\
\hline Glucose (mg/dL) & $93.14 \pm 8.62$ & $93.09 \pm 19.79$ & $94.14 \pm 14.57$ & $89.00 \pm 11.38^{*}$ & 0.188 \\
\hline $\mathrm{TG}(\mathrm{mg} / \mathrm{dL})$ & $109.14 \pm 39.31$ & $86.05 \pm 34.16^{*}$ & $109.90 \pm 44.72$ & $110.67 \pm 50.38^{\#}$ & $0.017^{\#}$ \\
\hline LDLC (mg/dL) & $127.68 \pm 31.69$ & $121.36 \pm 33.07$ & $146.00 \pm 38.56$ & $144.57 \pm 29.46$ & 0.106 \\
\hline HDLC (mg/dL) & $48.82 \pm 7.77$ & $52.18 \pm 6.46^{*}$ & $54.48 \pm 15.00$ & $54.95 \pm 15.57$ & 0.336 \\
\hline $\mathrm{TC}(\mathrm{mg} / \mathrm{dL})$ & $198.36 \pm 32.76$ & $190.73 \pm 34.78$ & $222.57 \pm 39.48$ & $221.71 \pm 35.35$ & 0.080 \\
\hline TC/HDLC ratio & $4.14 \pm 0.85$ & $3.70 \pm 0.78^{*}$ & $4.35 \pm 1.24$ & $4.29 \pm 1.21^{\#}$ & $0.048^{\#}$ \\
\hline AI & $3.14 \pm 0.85$ & $2.70 \pm 0.78^{*}$ & $3.35 \pm 1.24$ & $3.29 \pm 1.21^{\#}$ & $0.048^{\#}$ \\
\hline
\end{tabular}

Data are mean \pm SD. CON, continuous walking; INT, intermittent walking; TG, triglyceride; LDLC, low-density lipoprotein cholesterol; HDLC, high-density lipoprotein cholesterol; TC, total cholesterol; AI, atherosclerogenic index. ${ }^{*} p<0.05$, significantly different from before exercise program (two-way ANOVA). ${ }^{\#} p<0.05$, significantly different from the CON group (two-way ANOVA).

TABle 3: Heart rate, heart rate variability, and blood pressure of participants before and after the exercise program.

\begin{tabular}{|c|c|c|c|c|c|}
\hline & \multicolumn{2}{|c|}{ CON group $(n=22)$} & \multicolumn{2}{|c|}{ INT group $(n=21)$} & \multirow{2}{*}{$p$ value } \\
\hline & Before & After & Before & After & \\
\hline HR (beats/min) & $63.08 \pm 8.87$ & $63.91 \pm 9.38$ & $63.83 \pm 5.92$ & $60.44 \pm 6.38^{* \#}$ & $0.014^{\#}$ \\
\hline SDNN (ms) & $47.41 \pm 21.54$ & $36.58 \pm 15.40^{*}$ & $47.96 \pm 34.84$ & $49.85 \pm 26.70^{\#}$ & $0.016^{\#}$ \\
\hline RMSSD (ms) & $49.60 \pm 37.05$ & $34.02 \pm 24.97^{*}$ & $47.08 \pm 59.92$ & $51.59 \pm 44.77^{\#}$ & $0.018^{\#}$ \\
\hline $\mathrm{TP}\left(\mathrm{ms}^{2}\right)$ & $2579.75 \pm 2405.32$ & $1577.64 \pm 1710.37$ & $3233.71 \pm 6002.76$ & $2891.53 \pm 3578.45$ & 0.086 \\
\hline VLF power $\left(\mathrm{ms}^{2}\right)$ & $1218.70 \pm 1487.64$ & $765.12 \pm 685.81$ & $1038.63 \pm 780.51$ & $943.27 \pm 863.62$ & 0.358 \\
\hline $\mathrm{LF}$ power $\left(\mathrm{ms}^{2}\right)$ & $256.73 \pm 289.64$ & $204.92 \pm 204.83$ & $524.56 \pm 836.79$ & $442.87 \pm 600.78$ & 0.237 \\
\hline LFnu power & $28.91 \pm 18.16$ & $37.20 \pm 20.52$ & $41.97 \pm 16.65$ & $30.55 \pm 13.46^{* \#}$ & $0.043^{\#}$ \\
\hline $\mathrm{HF}$ power $\left(\mathrm{ms}^{2}\right)$ & $787.38 \pm 806.58$ & $481.68 \pm 993.05$ & $1046.87 \pm 2586.93$ & $1135.71 \pm 2138.87$ & 0.081 \\
\hline HFnu power & $55.43 \pm 15.60$ & $48.81 \pm 16.94$ & $47.45 \pm 9.83$ & $54.92 \pm 13.27$ & 0.103 \\
\hline $\mathrm{LF} / \mathrm{HF}$ ratio & $0.65 \pm 0.63$ & $1.21 \pm 1.68$ & $0.94 \pm 0.51$ & $0.67 \pm 0.48$ & 0.160 \\
\hline SBP (mmHg) & $127.50 \pm 15.35$ & $125.82 \pm 12.28$ & $129.97 \pm 14.84$ & $125.79 \pm 15.03$ & 0.626 \\
\hline DBP (mmHg) & $76.79 \pm 8.42$ & $76.27 \pm 6.71$ & $74.87 \pm 7.00$ & $74.11 \pm 7.38$ & 0.550 \\
\hline $\mathrm{PP}(\mathrm{mmHg})$ & $50.71 \pm 12.20$ & $49.55 \pm 11.22$ & $55.10 \pm 11.06$ & $51.68 \pm 11.94^{*}$ & 0.646 \\
\hline MAP (mmHg) & $93.69 \pm 9.63$ & $92.79 \pm 7.23$ & $93.24 \pm 8.89$ & $91.34 \pm 8.94$ & 0.531 \\
\hline RPP (mmHgbpm) & $8058.96 \pm 1609.64$ & $8058.35 \pm 1499.66$ & $8250.85 \pm 827.37$ & $7580.20 \pm 1043.28^{* \#}$ & $0.049^{\#}$ \\
\hline
\end{tabular}

Data are mean \pm SD. CON, continuous walking; INT, intermittent walking; HR, heart rate; SDNN, standard deviation of the NN intervals; RMSSD, root mean square of successive differences; TP, total power; VLF, very low frequency; LF, low frequency; HF, high frequency; SBP, systolic blood pressure; DBP, diastolic blood pressure; PP, pulse pressure; MAP, mean arterial pressure; RPP, rate-pressure product. ${ }^{*} p<0.05$, significantly different from before exercise program (two-way ANOVA). ${ }^{\#} p<0.05$, significantly different from CON group (two-way ANOVA).

that is, an increase in SDNN and RMSDD [46]. SDNN estimates variability due to both sympathetic and parasympathetic contributions and is highly correlated with LF power, especially amid $24 \mathrm{~h}$ recordings. The greater SDNN value predicts both lower morbidity and mortality [47]. RMSSD estimates vagally mediated changes reflected in HRV, and the greater RMSSD value is associated with lower scores on a risk inventory of sudden unexplained death [47]. Our results suggest that, with intermittent walking, parasympathetic (vagal) activity may be enhanced, leading to increased HRV, which is associated with the reduced risk of physiological and psychological conditions, that is, DM, CVD, inflammation, obesity, and psychiatric disorders [48]. Our observations are in agreement with a study by Gomes et al. [49] that found 8 weeks of regular low-intensity exercise enhanced cardiac structure and function, decreased oxidative stress, and conserved antioxidant enzyme activity amid rats with aortic stenosis-induced heart failure. These results reveal correlations among oxidative stress, cardiac function, and cardiac autonomic function. In addition, Thiyagarajan et al. [50] explored the association between HRV and elevated oxidative stress in diseases such as hypertension and subsequently found that increased oxidative stress is associated with decreased cardiovagal modulation. Accordingly, it is highly likely that alleviating oxidative stress following regular exercise would lead to improved cardiac autonomic function [51].

3.8. Regular Low-Intensity Exercise and Metabolic Profile. Fuller et al. [52] reported that a single session of low-intensity exercise induces rapid and robust shifts in mitochondrial and peroxisomal metabolism in the liver and skeletal muscle of healthy mice. Furthermore, 6 weeks of exercise training at that intensity restricted weight gain, increased muscle glycogen, and decreased TGs, though it did not alter substrate oxidation pathways. A previous study on obese populations by Campbell et al. [53] administered 12 weeks of continuous and intermittent walking exercise and reported that very low-density lipoprotein cholesterol decreased significantly $(-0.41 \mathrm{mM} / \mathrm{L})$ over time in the intermittent group, without any significant changes in TC, TG, HDLC, LDLC, and coronary risk ratio in both the continuous and intermittent walking groups. That study suggested 
that improvements in blood lipids as a result of exercise may be due to greater lipoprotein lipase activity [53]. As compared with that study, our study differed in some particulars, such as age and underlying diseases of participants, exercise frequency, duration, and mode. Hence, our results primarily demonstrate that serum HDLC increased significantly $(+3.4 \mathrm{mg} / \mathrm{dL})$. Serum TGs $(-23.1 \mathrm{mg} / \mathrm{dL})$, TC/HDLC ratio $(-0.4)$, and AI (-0.4) decreased significantly after continuous walking, and plasma glucose decreased significantly $(-5.1 \mathrm{mg} / \mathrm{dL})$ following intermittent walking. Moreover, the intergroup analysis demonstrated that serum TG, TC/ HDLC ratio, and AI were significantly lower in participants practicing continuous walking compared with those practicing intermittent walking.

Other related studies on continuous, low-intensity exercise have been reported. Paoli et al. [54] conducted a study on middle-aged overweight men and observed that lowintensity circuit training resulted in decreased TG $(-16.2 \mathrm{mg} / \mathrm{dL})$, TC $(-6 \mathrm{mg} / \mathrm{dL})$, LDLC $(-3.2 \mathrm{mg} / \mathrm{dL})$, and apolipoprotein B/A1 $(-0.06)$. Besides that, there was a greater reduction in SBP $(-11 \mathrm{mmHg})$ compared with highintensity circuit $(-7 \mathrm{mmHg})$ and endurance training ( $-5 \mathrm{mmHg})$. In addition, Gaesser and Rich [55] determined the effect of low-intensity cycling exercise over 18 weeks in healthy males and discovered a significant reduction in $\% \mathrm{BF}$, amounting to an average fat loss of approximately $1.35 \mathrm{~kg}$. However, that study did not observe a significant alteration in blood lipids (i.e., TC, TG, HDLC, LDLC, TC/HDLC, and HDLC/LDLC). Teodoro et al. [56] showed that 8 weeks of low-intensity cardiovascular exercise increased antioxidant enzyme activities, decreased lipid hydroperoxide and protein carbonyl formation, and decreased the number of atherosclerotic lesions in mice with atherosclerosis. This study emphasized the links between oxidative stress, metabolic abnormality, and the development of atherosclerosis.

A study on mild spinal muscular atrophy-like mice, which administered low-intensity running for 10 months, revealed that exercise significantly improved lipid metabolism and glucose homeostasis and enhanced oxygen consumption $\left(\mathrm{VO}_{2}\right)$ efficiency [57]. The beneficial effects of low-intensity exercise that differ from other exercises may be related to the differential use of energetic substrates linked to exercise intensity [58]. Regular low-intensity exercise can shift metabolism from $\beta$-oxidation toward the use of carbohydrate oxidation. This exercise-induced energy pathway modifies lipid and carbohydrate metabolism. Moreover, regular low-intensity exercise increases the expression of mitochondrial respiratory chain complexes, resulting in enhanced mitochondrial oxidative capacity. It also enhances glucose tolerance and is associated with a decrease in mRNA expression of lipogenic enzymes in fat tissues, hence establishing enhanced whole-body metabolism [57].

3.9. Regular Low-Intensity Exercise and Body Composition. Results of Going et al. [59] demonstrated a strong relationship between \%BF and chronic disease risk factors, that is, BP, lipids and lipoproteins, glucose, insulin, and C-reactive protein levels. $\% \mathrm{BF}$ has also been shown to be a risk factor for CVD and metabolic syndrome in both men and women, independent of BMI [60]. Targeted reduction of $\%$ BF may improve cardiovascular outcomes [61]. To our knowledge, few studies have investigated the effects of intermittent, low-intensity exercise, and most studies have incorporated continuous, low-intensity exercise. In this study, we observed significant changes in body composition only in the intermittent walking group, except for a decrease in hip circumference $(-1.3 \mathrm{~cm})$ in the continuous walking group. These changes included decreased FM $(-4.1 \mathrm{~kg}), \% \mathrm{BF}$ $(-5.8 \%)$, visceral fat level $(-1.5), \mathrm{HC}(-5.2 \mathrm{~cm})$, increased $\%$ FFM $(+5.9 \%)$, and \%body water $(+4.3 \%)$, although BMI did not reach a significant decrease $\left(-1.6 \mathrm{~kg} / \mathrm{m}^{2}\right)$. Our observations are similar, yet with mostly to a greater extent as compared to previous reports. Findings from a study by Chiu et al. [62] revealed that regular low-intensity exercise at 40 to $50 \%$ heart rate reserve resulted in significantly decreased BM $(-2.9 \mathrm{~kg}), \mathrm{BMI}\left(-1 \mathrm{~kg} / \mathrm{m}^{2}\right)$, WC $(-5.3 \mathrm{~cm}), \mathrm{W} / \mathrm{H}$ ratio $(-0.04), \% \mathrm{BF}(-1.70 \%)$, and $\mathrm{FM}(-2.6 \mathrm{~kg})$ in sedentary obese college students. Additionally, Suzuki et al. [63] reported on low-intensity cycling exercise at $40 \%$ of maximum $\mathrm{VO}_{2}\left(\mathrm{VO}_{2 \max }\right)$ for 12 weeks, which resulted in decreased BMI $(-1.9 \%)$ and \%BF $(-2.0 \%)$ in sedentary young females. Further study on patients with metabolic syndrome by Dumortier et al. [64] observed that low-intensity endurance exercise for 2 months improved lipid oxidation $(+68.5 \mathrm{mg} /$ min) and body composition, that is, FM $(-1.6 \mathrm{~kg})$, WC $(-3.5 \mathrm{~cm})$, and $\mathrm{HC}(-2.2 \mathrm{~cm})$. What is more, Lazzer et al. [65] observed that BM (-7.4\%), BMI (-7.7\%), and FM $(-10.1 \%)$ significantly decreased in severely obese adolescents performing low-intensity treadmill exercise at $40 \%$ $\mathrm{VO}_{2 \max }$ for 3 weeks. In that study, the authors also suggested that low-intensity exercise favors fat oxidation and is more feasible and acceptable than high-intensity exercise for obese adolescents.

3.10. Regular Low-Intensity Exercise and BP. Clinical data have provided an association between oxidative stress and hypertension [66]. Ye et al. [67] suggested that reactive oxygen species (ROS) may elevate BP by stimulating the sympathetic nervous system (SNS). Because NO exerts tonic inhibition on central SNS activity, increased ROS production may trigger the SNS through oxidation/inactivation of NO. Hua et al. [68] quantified the effects of a 12-week homebased low-intensity walking exercise program in hypertensive men and women and showed a training effect in which SBP, DBP, and the R-R interval decreased significantly, and spontaneous baroreflex sensitivity increased significantly in the exercising group.

In this study, the decline in BP was not statistically significant. SBP and DBP decreased by approximately 2 and $0.5 \mathrm{mmHg}$ in the continuous walking group, respectively, whereas in the intermittent walking group, SBP and DBP decreased by approximately 4 and $0.5 \mathrm{mmHg}$, respectively. A study by Lu et al. [26] conducted in adults with hypertension and prehypertension revealed that regular sessions of slow walking for 50-60 min/day at 5-7 times/week significantly lowered BP and HR. In that study, the authors suggested that 
$\beta$-endorphin could act as a buffer against exercise-related sympathetic excitation and cardiovascular overload, which may play a role in pressure-lowering and negative chronotropic effects. Motoyama et al. [69] observed that resting SBP $(-15 \mathrm{mmHg})$, MAP $(-11 \mathrm{mmHg})$, and DBP $(-9 \mathrm{mmHg})$ decreased significantly following 3 months of low-intensity treadmill exercise for 30 min, 3-6 times a week in elderly hypertensive patients. The slight decrease in SBP and DBP without major significance in our study compared with previous reports may be due to a difference in duration, frequency of exercise, and training period. Nevertheless, a greater reduction in SBP in the intermittent walking group resulted in decreased PP in this group after exercise program participation. Moreover, with a significantly lower resting $\mathrm{HR}$ and greater reduction in SBP in the intermittent walking group, RPP was expressed to be lower in participants who partook in intermittent walking. Normally, BP control is also associated with other influencing factors, including baroreflex sensitivity, endothelial function, and the renin-angiotensin system [70]. It seems that those factors need to be correspondingly improved for BP lowering since they appear to be intensity- and mode-dependent [71].

Notably, it has been estimated that a $2 \mathrm{mmHg}$ decrease in SBP would result in a $6 \%$ overall reduction in mortality due to stroke, a $4 \%$ reduction in mortality due to coronary heart disease, and a 3 to $4 \%$ reduction in all-cause mortality [72]. Accordingly, our findings emphasize the effects of long-term regular walking as exercise therapy in older people with hypertension. Furthermore, the results encourage the Guideline Recommendations of the American College of Cardiology and American Heart Association in that regular exercise should be prescribed for the prevention and management of hypertension [73].

3.11. Study Limitations. This study has certain limitations. Most of the participants received concomitant medications, such as calcium channel blockers, angiotensin receptor blockers, angiotensin converting enzyme inhibitors, betablockers, or diuretics, before and during the exercise program. Some findings may be partly affected by those treatments, although there was no change in medications administered during the study. In addition, few studies have evaluated the effects of long-term regular low-intensity exercise on BP and the relevant factors. Also, no study has compared continuous to intermittent low-intensity exercise in older populations with hypertension. Consequently, our discussion is based on closely related evidence. This study program was designed as a home-based exercise that afforded participants flexibility in terms of performing the exercise. Nevertheless, good adherence to and compliance with the exercise programs was reported.

\section{Conclusions}

This study provides evidence that practicing long-term regular continuous and intermittent walking may hold potential for increasing antioxidant and decreasing lipid peroxidation, thus alleviating oxidative stress in older participants with hypertension. Moreover, the participants practicing continuous walking exhibited greater improvement in metabolic profile, while the participants practicing intermittent walking showed greater improvement in cardiac autonomic function.

\section{Data Availability}

No data were used to support this study.

\section{Conflicts of Interest}

The authors declare that there are no conflicts of interest regarding the publication of this paper.

\section{Acknowledgments}

This work was financially supported by the Burapha University through the National Research Council of Thailand (Grant no. 230/2561). This work was supported by the Burapha University Research and Development Fund and the Exercise and Nutrition Innovation and Sciences Research Unit, Burapha University, Thailand.

\section{References}

[1] N. D. L. Fisher and G. Curfman, "Hypertension-a public health challenge of global proportions," JAMA, vol. 320, no. 17, pp. 1757-1759, 2018.

[2] A. Benetos, M. Petrovic, and T. Strandberg, "Hypertension management in older and frail older patients," Circulation Research, vol. 124, no. 7, pp. 1045-1060, 2019.

[3] B. M. Egan, S. E. Kjeldsen, G. Grassi, M. Esler, and G. Mancia, "The global burden of hypertension exceeds 1.4 billion people: should a systolic blood pressure target below 130 become the universal standard?" Journal of Hypertension, vol. 37, no. 6, pp. 1148-1153, 2019.

[4] K. T. Mills, J. D. Bundy, T. N. Kelly et al., "Global disparities of hypertension prevalence and control: a systematic analysis of population-based studies from 90 countries," Circulation, vol. 134, no. 6, pp. 441-450, 2016.

[5] J. F. Machi, N. Bernardes, C. Mostarda et al., "Walking promotes metabolic and baroreflex sensitivity improvement in fructose-fed male rats," European Journal of Applied Physiology, vol. 113, no. 1, pp. 41-49, 2013.

[6] T. Tofas, D. Draganidis, C. K. Deli, K. Georgakouli, I. G. Fatouros, and A. Z. Jamurtas, "Exercise-induced regulation of redox status in cardiovascular diseases: the role of exercise training and detraining," Antioxidants, vol. 9, no. 1, p. 13, 2019.

[7] W. Qu, G.-L. Du, B. Feng, and H. Shao, "Effects of oxidative stress on blood pressure and electrocardiogram findings in workers with occupational exposure to lead," Journal of International Medical Research, vol. 47, no. 6, pp. 2461-2470, 2019.

[8] K. Hyodo, K. Suwabe, D. Yamaguchi, H. Soya, and T. Arao, "Comparison between the effects of continuous and intermittent light-intensity aerobic dance exercise on mood and executive functions in older adults," Frontiers in Aging Neuroscience, vol. 13, Article ID 723243, 2021.

[9] H. Patel, H. Alkhawam, R. Madanieh, N. Shah, C. E. Kosmas, and T. J. Vittorio, "Aerobicvsanaerobic exercise training 
effects on the cardiovascular system," World Journal of Cardiology, vol. 9, no. 2, pp. 134-138, 2017.

[10] S. Sabapathy, R. A. Kingsley, D. A. Schneider, L. Adams, and N. R. Morris, "Continuous and intermittent exercise responses in individuals with chronic obstructive pulmonary disease," Thorax, vol. 59, no. 12, pp. 1026-1031, 2004.

[11] M. Izquierdo, R. A. Merchant, J. E. Morley et al., "International exercise recommendations in older adults (ICFSR): expert consensus guidelines," The Journal of Nutrition, Health \& Aging, vol. 25, no. 7, pp. 824-853, 2021.

[12] M. G. Rodriguez-Hernandez and D. W. Wadsworth, "The effect of 2 walking programs on aerobic fitness, body composition, and physical activity in sedentary office employees," PLoS One, vol. 14, no. 1, Article ID e0210447, 2019.

[13] H. Cerda-Kohler, Y. Pullin, and J. Cancino-López, "Effects of continuous and intermittent endurance exercise in autonomic balance, rating perceived exertion and blood lactate levels in healthy subjects," Apunts. Medicina de l'Esport, vol. 50, no. 185, pp. 29-34, 2015.

[14] K. Seidelin, M. Nyberg, P. Piil, N. R. Jørgensen, Y. Hellsten, and J. Bangsbo, "Adaptations with intermittent exercise training in post- and premenopausal women," Medicine \& Science in Sports \& Exercise, vol. 49, no. 1, pp. 96-105, 2017.

[15] J. X. Fan, L. Kowaleski-Jones, and M. Wen, "Walking or dancing: patterns of physical activity by cross-sectional age among U.S. women," Journal of Aging and Health, vol. 25, no. 7, pp. 1182-1203, 2013.

[16] S. Mandini, F. Conconi, E. Mori, J. Myers, G. Grazzi, and G. Mazzoni, "Walking and hypertension: greater reductions in subjects with higher baseline systolic blood pressure following six months of guided walking," PeerJ, vol. 6, Article ID e5471, 2018.

[17] L. He, W. r. Wei, and Z. Can, "Effects of 12-week brisk walking training on exercise blood pressure in elderly patients with essential hypertension: a pilot study," Clinical and Experimental Hypertension, vol. 40, no. 7, pp. 673-679, 2018.

[18] G. A. Trevizani, T. Peçanha, O. Nasario-Junior, J. M. Vianna, L. P. Silva, and J. Nadal, "Cardiac autonomic responses after resistance exercise in treated hypertensive subjects," Frontiers in Physiology, vol. 6, p. 258, 2015.

[19] C. P. Stewart, P. Christian, L. S. F. Wu, S. C. LeClerq, S. K. Khatry, and K. P. West Jr., "Prevalence and risk factors of elevated blood pressure, overweight, and dyslipidemia in adolescent and young adults in rural Nepal," Metabolic Syndrome and Related Disorders, vol. 11, no. 5, pp. 319-328, 2013.

[20] R. P. Hernández-Torres, A. Ramos-Jiménez, P. V. TorresDurán, J. Romero-Gonzalez, D. Mascher, and C. PosadasRomero, "Effects of single sessions of low-intensity continuous and moderate-intensity intermittent exercise on blood lipids in the same endurance runners," Journal of Science and Medicine in Sport, vol. 12, no. 2, pp. 323-331, 2009.

[21] B. Williams, G. Mancia, W. Spiering, E. Agabiti Rosei, M. Azizi, and M. Burnier, "2018 ESC/ESH Guidelines for the management of arterial hypertension," European Heart Journal, vol. 39, no. 33, pp. 3021-3104, 2018.

[22] C. Gaucher, A. Boudier, J. Bonetti, I. Clarot, P. Leroy, and M. Parent, "Glutathione: antioxidant properties dedicated to nanotechnologies," Antioxidants, vol. 7, no. 5, p. 62, 2018.

[23] S. Nakmareong, U. Kukongviriyapan, P. Pakdeechote et al., "Tetrahydrocurcumin alleviates hypertension, aortic stiffening and oxidative stress in rats with nitric oxide deficiency," Hypertension Research, vol. 35, no. 4, pp. 418-425, 2012.

[24] G. Jan-On, W. Sangartit, P. Pakdeechote et al., "Virgin rice bran oil alleviates hypertension through the upregulation of
eNOS and reduction of oxidative stress and inflammation in L-NAME-induced hypertensive rats," Nutrition, vol. 69, Article ID 110575, 2020.

[25] U. Kukongviriyapan, V. Kukongviriyapan, P. Pannangpetch et al., "Mamao pomace extract alleviates hypertension and oxidative stress in nitric oxide deficient rats," Nutrients, vol. 7, no. 8, pp. 6179-6194, 2015.

[26] Q. Lu, S.-m. Wang, Y.-x. Liu et al., "Low-intensity walking as mild medication for pressure control in prehypertensive and hypertensive subjects: how far shall we wander?" Acta Pharmacologica Sinica, vol. 40, no. 8, pp. 1119-1126, 2019.

[27] K. S. Hall, C. A. Howe, S. R. Rana, C. L. Martin, and M. C. Morey, "METs and accelerometry of walking in older adults," Medicine \& Science in Sports \& Exercise, vol. 45, no. 3, pp. 574-582, 2013.

[28] B. E. Ainsworth, W. L. Haskell, M. C. Whitt, M. L. Irwin, A. M. Swartz, and S. J. Strath, "Compendium of physical activities: an update of activity codes and MET intensities," Medicine \& Science in Sports \& Exercise, vol. 32, no. 9 suppl, pp. S498-S516, 2000.

[29] Y. S. Kang, D. Seong, J. C. Kim, and S. H. Kim, "Low-intensity exercise training additionally increases mitochondrial dynamics caused by high-fat diet (HFD) but has no additional effect on mitochondrial biogenesis in fast-twitch muscle by HFD," International Journal of Environmental Research and Public Health, vol. 17, no. 15, p. 5461, 2020.

[30] I. Liguori, G. Russo, F. Curcio et al., "Oxidative stress, aging, and diseases," Clinical Interventions in Aging, vol. 13, pp. 757-772, 2018.

[31] T. Münzel, G. G. Camici, C. Maack, N. R. Bonetti, V. Fuster, and J. C. Kovacic, "Impact of oxidative stress on the heart and vasculature: part 2 of a 3-part series," Journal of the American College of Cardiology, vol. 70, no. 2, pp. 212-229, 2017.

[32] B. L. Tan, M. E. Norhaizan, W.-P.-P. Liew, and H. Sulaiman Rahman, "Antioxidant and oxidative stress: a mutual interplay in age-related diseases," Frontiers in Pharmacology, vol. 9, p. 1162, 2018.

[33] H. Tomiyama, K. Shiina, C. Matsumoto-Nakano, T. Ninomiya, S. Komatsu, and K. Kimura, "The contribution of inflammation to the development of hypertension mediated by increased arterial stiffness," Journal of American Heart Association, vol. 6, no. 7, Article ID e005729, 2017.

[34] A. Nguyen, N. Duquette, M. Mamarbachi, and E. Thorin, "Epigenetic regulatory effect of exercise on glutathione peroxidase 1 expression in the skeletal muscle of severely dyslipidemic mice," PLoS One, vol. 11, no. 3, Article ID e0151526, 2016.

[35] M. S. d. S. Fernandes, L. d. L. d. S. e. Silva, M. S. Kubrusly et al., "Aerobic exercise training exerts beneficial effects upon oxidative metabolism and non-enzymatic antioxidant defense in the liver of leptin deficiency mice," Frontiers in Endocrinology, vol. 11, Article ID 588502, 2020.

[36] M. Nyberg, S. P. Mortensen, H. Cabo, M.-C. Gomez-Cabrera, J. Viña, and Y. Hellsten, "Roles of sedentary aging and lifelong physical activity in exchange of glutathione across exercising human skeletal muscle," Free Radical Biology and Medicine, vol. 73, pp. 166-173, 2014.

[37] P. C. Douris, A. S. Elokda, J. P. Handrakis et al., "Martial art training enhances the glutathione antioxidant system in middle-aged adults," The Journal of Strength \& Conditioning Research, vol. 23, no. 5, pp. 1518-1523, 2009.

[38] A. S. Elokda and D. H. Nielsen, "Effects of exercise training on the glutathione antioxidant system," European Journal of Cardiovascular Prevention \& Rehabilitation, vol. 14, no. 5, pp. 630-637, 2007. 
[39] M. A. Bouzid, O. Hammouda, R. Matran, S. Robin, and C. Fabre, "Influence of physical fitness on antioxidant activity and malondialdehyde level in healthy older adults," Applied Physiology Nutrition and Metabolism, vol. 40, no. 6, pp. 582-589, 2015.

[40] T. L. Merry and M. Ristow, "Do antioxidant supplements interfere with skeletal muscle adaptation to exercise training?" The Journal of Physiology, vol. 594, no. 18, pp. 5135-5147, 2016.

[41] A. Linke, V. Adams, P. C. Schulze et al., "Antioxidative effects of exercise training in patients with chronic heart failure," Circulation, vol. 111, no. 14, pp. 1763-1770, 2005.

[42] C. Simioni, G. Zauli, A. M. Martelli et al., "Oxidative stress: role of physical exercise and antioxidant nutraceuticals in adulthood and aging," Oncotarget, vol. 9, no. 24, pp. 17181-17198, 2018.

[43] F. R. Roque, A. M. Briones, A. B. García-Redondo et al., "Aerobic exercise reduces oxidative stress and improves vascular changes of small mesenteric and coronary arteries in hypertension," British Journal of Pharmacology, vol. 168, no. 3, pp. 686-703, 2013.

[44] K. Klarod, P. Hongsprabhas, T. Samangeon, K. Hongto, R. Tangrassameeprasert, and P. Boonsiri, "Effect of low-intensity exercise on oxidative stress, inflammatory and nutritional responsiveness in sedentary people," J Nutr Health Food Sci, vol. 5, no. 2, pp. 1-7, 2017.

[45] B. K. Koichubekov, M. A. Sorokina, Y. M. Laryushina, L. G. Turgunova, and I. V. Korshukov, "Nonlinear analyses of heart rate variability in hypertension," Annales de Cardiologie et d'Angeiologie, vol. 67, no. 3, pp. 174-179, 2018.

[46] C. S. Mclachlan, R. Ocsan, I. Spence et al., "Increased total heart rate variability and enhanced cardiac vagal autonomic activity in healthy humans with sinus bradycardia," Baylor University Medical Center Proceedings, vol. 23, no. 4, pp. 368-370, 2010.

[47] F. Shaffer and J. P. Ginsberg, "An Overview of heart rate variability metrics and norms," Frontiers in Public Health, vol. 5, p. 258, 2017.

[48] H. A. Young and D. Benton, "Heart-rate variability: a biomarker to study the influence of nutrition on physiological and psychological health?" Behavioural Pharmacology, vol. 29, pp. 140-151, 2018.

[49] M. J. Gomes, P. F. Martinez, D. H. Campos et al., "Beneficial effects of physical exercise on functional capacity and skeletal muscle oxidative stress in rats with aortic stenosis-induced heart failure," Oxidative Medicine and Cellular Longevity, vol. 2016, Article ID 8695716, 12 pages, 2016.

[50] R. Thiyagarajan, P. Pal, G. K. Pal et al., "Cardiovagal modulation, oxidative stress, and cardiovascular risk factors in prehypertensive subjects: cross-sectional study," American Journal of Hypertension, vol. 26, no. 7, pp. 850-857, 2013.

[51] R. Thiyagarajan, S. K. Subramanian, N. Sampath et al., "Association between cardiac autonomic function, oxidative stress and inflammatory response in impaired fasting glucose subjects: cross-sectional study," PLoS One, vol. 7, no. 7, Article ID e41889, 2012.

[52] S. E. Fuller, T. Y. Huang, J. Simon, H. M. Batdorf, N. M. Essajee, and M. C. Scott, "Low-intensity exercise induces acute shifts in liver and skeletal muscle substrate metabolism but not chronic adaptations in tissue oxidative capacity," Journal of Applied Physiology, vol. 127, no. 1, pp. 143-156, 2019.

[53] L. Campbell, K. Wallman, and D. Green, "The effects of intermittent exercise on physiological outcomes in an obese population: continuous versus interval walking," Journal of Sports Science \& Medicine, vol. 9, no. 1, pp. 24-30, 2010.

[54] A. Paoli, Q. F. Pacelli, T. Moro et al., "Effects of high-intensity circuit training, low-intensity circuit training and endurance training on blood pressure and lipoproteins in middle-aged overweight men," Lipids in Health and Disease, vol. 12, no. 1, p. 131, 2013.

[55] G. A. Gaesser and R. G. Rich, "Effects of high- and low-intensity exercise training on aerobic capacity and blood lipids," Medicine \& Science in Sports \& Exercise, vol. 16, no. 3, pp. 269-274, 1984.

[56] B. G. Teodoro, A. o. n. J. e. Natali, S. i. 1. A. T. Fernandes et al., "Improvements of atherosclerosis and hepatic oxidative stress are independent of exercise intensity in LDLr-/- mice," Journal of Atherosclerosis and Thrombosis, vol. 19, no. 10, pp. 904-911, 2012.

[57] L. Houdebine, D. D’Amico, J. Bastin et al., "Low-intensity running and high-intensity swimming exercises differentially improve energy metabolism in mice with mild spinal muscular atrophy," Frontiers in Physiology, vol. 10, p. 1258, 2019.

[58] F. Chali, C. Desseille, L. Houdebine et al., "Long-term exercise-specific neuroprotection in spinal muscular atrophylike mice," The Journal of Physiology, vol. 594, no. 7, pp. 1931-1952, 2016.

[59] S. B. Going, T. G. Lohman, E. C. Cussler, D. P. Williams, J. A. Morrison, and P. S. Horn, "Percent body fat and chronic disease risk factors in U.S. children and youth," American Journal of Preventive Medicine, vol. 41, no. 4 Suppl 2, pp. S77-S86, 2011.

[60] H.-H. Chuang, W.-C. Li, B.-F. Sheu et al., "Correlation between body composition and risk factors for cardiovascular disease and metabolic syndrome," BioFactors, vol. 38, no. 4, pp. 284-291, 2012.

[61] Z. Zhuang, M. Yao, J. Y. Y. Wong, Z. Liu, and T. Huang, "Shared genetic etiology and causality between body fat percentage and cardiovascular diseases: a large-scale genome-wide cross-trait analysis," BMC Medicine, vol. 19, no. 1, p. 100, 2021.

[62] C.-H. Chiu, M.-C. Ko, L.-S. Wu et al., "Benefits of different intensity of aerobic exercise in modulating body composition among obese young adults: a pilot randomized controlled trial," Health and Quality of Life Outcomes, vol. 15, no. 1, p. 168, 2017.

[63] S. Suzuki, G. Urata, Y. Ishida, H. Kanehisa, and M. Yamamura, "Influences of low intensity exercise on body composition, food intake and aerobic power of sedentary young females," Applied Human Science Journal of Physiological Anthropology, vol. 17, no. 6, pp. 259-266, 1998.

[64] M. Dumortier, F. Brandou, A. Perez-Martin, C. Fedou, J. Mercier, and J. Brun, "Low intensity endurance exercise targeted for lipid oxidation improves body composition and insulin sensitivity in patients with the metabolic syndrome," Diabetes and Metabolism, vol. 29, no. 5, pp. 509-518, 2003.

[65] S. Lazzer, C. Lafortuna, C. Busti, R. Galli, F. Agosti, and A. Sartorio, "Effects of low- and high-intensity exercise training on body composition and substrate metabolism in obese adolescents," Journal of Endocrinological Investigation, vol. 34, no. 1, pp. 45-52, 2011.

[66] M. Korsager Larsen and V. V. Matchkov, "Hypertension and physical exercise: the role of oxidative stress," Medicina, vol. 52, no. 1, pp. 19-27, 2016.

[67] S. Ye, H. Zhong, V. M. Campese, and V. M. Campese, "Oxidative stress mediates the stimulation of sympathetic nerve activity in the phenol renal injury model of hypertension," Hypertension, vol. 48, no. 2, pp. 309-315, 2006. 
[68] L. P. T. Hua, C. A. Brown, S. J. M. Hains, M. Godwin, and J. L. Parlow, "Effects of low-intensity exercise conditioning on blood pressure, heart rate, and autonomic modulation of heart rate in men and women with hypertension," Biological Research For Nursing, vol. 11, no. 2, pp. 129-143, 2009.

[69] M. Motoyama, Y. Sunami, F. Kinoshita et al., "Blood pressure lowering effect of low intensity aerobic training in elderly hypertensive patients," Medicine \& Science in Sports \& Exercise, vol. 30, no. 6, pp. 818-823, 1998.

[70] M. M. Asemu, A. W. Yalew, N. D. Kabeta, and D. Mekonnen, "Prevalence and risk factors of hypertension among adults: a community based study in Addis Ababa, Ethiopia," PLoS One, vol. 16, no. 4, Article ID e0248934, 2021.

[71] E. Morvan, N. E. A. Lima, J. F. Machi et al., "Metabolic, hemodynamic and structural adjustments to low intensity exercise training in a metabolic syndrome model," Cardiovascular Diabetology, vol. 12, no. 1, p. 89, 2013.

[72] A. V. Chobanian, G. L. Bakris, H. R. Black et al., "Seventh report of the joint national committee on prevention, detection, evaluation, and treatment of high blood pressure," Hypertension, vol. 42, no. 6, pp. 1206-1252, 2003.

[73] P. K. Whelton, R. M. Carey, W. S. Aronow, D. E. Casey Jr., K. J. Collins, and C. Dennison Himmelfarb, "2017 ACC/AHA/ AAPA/ABC/ACPM/AGS/APhA/ASH/ASPC/NMA/PCNA guideline for the prevention, detection, evaluation, and management of high blood pressure in adults: executive summary: a report of the American college of Cardiology/ American heart association task force on clinical practice guidelines," Hypertension, vol. 71, no. 6, pp. 1269-1324, 2018. 